BU-HEP-BUHEP-99-26

DOE/ER/40561-76-INT99

\title{
Solving the Hierarchy Problem with Noncompact Extra Dimensions
}

\author{
Andrew G. Cohen ${ }^{a}$ and David B. Kaplan ${ }^{b}$ * \\ ${ }^{a}$ Department of Physics, Boston University, Boston, MA 02215, USA \\ ${ }^{b}$ Institute for Nuclear Theory, 1550, University of Washington, Seattle, WA 98195-1550, USA
}

October 16, 1999

\begin{abstract}
We show that gravitational effects of global cosmic 3-branes can be responsible for compactification from six to four space-time dimensions, naturally producing the observed hierarchy between electroweak and gravitational forces. The finite radius of the transverse dimensions follows from Einstein's equation, and is exponentially large compared with the scales associated with the 3-brane. The space-time ends on a mild naked singularity at the boundary of the transverse dimensions; nevertheless unitary boundary conditions render the singularity harmless.
\end{abstract}

*cohen@bu.edu, dbkaplan@phys.washington.edu 


\section{Introduction}

The possibility that we are living in more than four dimensions has been considered sporadically by physicists since the time of Kaluza [1] and Klein [2, 3]. Although originally motivated by the possible unification of gravity with gauge forces, more recently there have been speculations that extra dimensions may also play an important role in addressing several other outstanding phenomenological problems - in particular, the smallness of the cosmological constant, the origin of the observed hierarchy between the $W$ mass and the Planck scale, the nature of flavor, the possible source of supersymmetry breaking, etc. In this paper we focus on the possibility that extra dimensions may provide a resolution of the hierarchy problem.

There have been two distinct approaches using extra dimensions to deal with the hierarchy problem. One relies on compact extra dimensions, curled up in such a way that 4-d phenomenology prevails. The other uses noncompact extra dimensions of infinite extent. The first category includes the "large extra dimension" approach, which postulates a fundamental scale $\Lambda \sim 10-100 \mathrm{TeV}$ along with Kaluza-Klein compactification at a large radius $R$ ( $\sim$ millimeters for two extra dimensions). The effective 4 -d Planck scale is then the fundamental scale $\Lambda$ times powers of $\Lambda R$ [4, 5, 6, 7, 8]. If $R$ is much larger than $\Lambda^{-1}$, the effective Planck scale will be much larger than the fundamental scale. This does not solve the hierarchy problem but rather reformulates it into a dynamical question: why is the radius $R$ of the extra dimensions so much larger than $1 / \Lambda$ ? Mechanisms exist which can answer this question [9]. Another possible hierarchy solution arising from compact extra dimensions is the model of ref. [10], where an exponential warp factor gives rise to the observed hierarchy, requiring an extra dimension only one or two orders of magnitude larger than the fundamental length scale. A dynamical mechanism to stabilize the radius is still needed, such as first suggested in [11, 12].

It has been shown that ordinary 4-d gravity can result from a theory with infinite extra dimensions, provided that there exists a graviton bound to our 4-d submanifold [13, 14, 15]. Such models do not in general solve the hierarchy problem. In refs. [16, 17] it was suggested that, since the wave function of the bound 4-d graviton falls off exponentially in the direction transverse to the 3-brane that binds it, the observed hierarchy of forces may be due to a displacement of our world from that 3-brane.

In this paper we offer an alternative where the hierarchy arises from finite, but noncompact extra dimensions. The phenomenology closely resembles the large extra dimension scenario [5, 6, 8], but provides a dynamical explanation for the large radius. However, unlike these earlier scenarios, the extra dimensions we consider form a non-compact space of exponentially large proper size, terminating at a singularity. The spacetime we analyze is the solution of Einstein's equation in the presence of a global cosmic string [18].

We begin by solving Einstein's equation for a global cosmic "string" in $d+2$ dimensions. By a "string" we will always mean a $(d-1)$-brane with 2 transverse spatial dimensions. We analyze scalar and gravitational waves in this background metric, and demonstrate that although the spacetime includes a naked singularity, it is sufficiently mild that unitary boundary conditions can be applied. We then display the hierarchy between gravitational 
and gauge forces, showing that it is not the result of any fine tuning, and does not lead to disagreement with gravitational force experiments. We conclude by discussing possible generalizations of our example.

\section{The metric about a global string}

A global cosmic string, or vortex, is a topologically stable scalar field configuration with nontrivial homotopy for some internal symmetry manifold. The simplest example arises in a scalar field theory with a spontaneously broken global $U(1)$ symmetry. If the expectation value of the scalar field in the ground state is $\langle\Phi\rangle=f$, a vortex with unit winding number corresponds to the field configuration $\Phi(r)=f(r) e^{i \theta}$, where $\lim _{r \rightarrow \infty} f(r)=f$. Unlike a vortex with the $U(1)$ gauged (such as the Abelian Higgs model), a global vortex has nonzero energy density outside its core, falling off as $1 / r^{2}$. It is therefore not surprising that, when gravity is included, a curvature singularity appears exponentially far from the core of the vortex, as was found in earlier work by the present authors for vortices in four spacetime dimensions 18 .

Generalization of this earlier work to branes in $d+2$ spacetime dimensions is straightforward. We assume that outside the core of a $(d-1)$-brane, the stress-energy tensor is that of a charged scalar field with Lagrange density円 $\mathcal{L}=-g^{\mu \nu} \partial_{\mu} \Phi^{*} \partial_{\nu} \Phi-V(\Phi)$ and expectation value

$$
\Phi=f^{d / 2} e^{i \theta}
$$

where $f$ has dimensions of mass. The scalar field eq. (11) is assumed to minimize the scalar potential $V(\Phi)$, and we tune the bulk cosmological constant to zero. We therefore look for a metric which has a $d$-dimensional Poincaré invariant "longitudinal" space, and rotational invariance in the transverse plane. The most general metric of this kind may be put in the form

$$
d s^{2}=\bar{A}(u)^{2} \eta_{a b} d x^{a} d x^{b}+\gamma^{2} \bar{B}(u)^{2}\left(d u^{2}+d \theta^{2}\right)
$$

where $x^{a}$ parameterizes the longitudinal $d$-dimensional space, $\eta_{a b}$ is the flat Minkowski metric, and $\{u, \theta\}$ are the transverse coordinates, with $\theta \in[0,2 \pi)$. The parameter $\gamma$ has dimensions of length, while $u, \theta$ and the functions $\bar{A}$ and $\bar{B}$ are dimensionless. We will make the somewhat perverse choice of placing the string core at large values of $u$, while the singularity will appear at $u=0$.

The non-zero components of the stress-energy tensor which follow from $\mathcal{L}$ and eq. (四) are:

$$
T_{j}^{i}=\delta_{j}^{i} \frac{f^{d}}{\gamma^{2} \bar{B}^{2}}, \quad T_{u}^{u}=\frac{f^{d}}{\gamma^{2} \bar{B}^{2}}, \quad T_{\theta}^{\theta}=-\frac{f^{d}}{\gamma^{2} \bar{B}^{2}} .
$$

\footnotetext{
${ }^{1}$ We adopt the mostly plus sign convention.
} 
The Einstein equations for this system are

$$
G_{\nu}^{\mu}=-\frac{1}{M_{d+2}^{d}} T_{\nu}^{\mu}
$$

where $M_{d+2}$ is the analogue of the Planck mass in $d+2$ dimensions.

On computing the Einstein tensor from the metric in eq. (2), we arrive at the three differential equations

$$
\begin{aligned}
(d-1)\left(\bar{A}^{\prime}\right)^{2}+\bar{A} \bar{A}^{\prime \prime} & =0 \\
\bar{A}\left(\bar{B}^{\prime}\right)^{2}+\bar{B}\left[d \bar{A}^{\prime} \bar{B}^{\prime}-d \bar{B} \bar{A}^{\prime \prime}-\bar{A} \bar{B}^{\prime \prime}\right] & =0 \\
\bar{A}\left(\bar{B}^{\prime}\right)^{2}-\bar{B}\left[d \bar{A}^{\prime} \bar{B}^{\prime}+\bar{A} \bar{B}^{\prime \prime}\right] & =\frac{2}{u_{0}} \bar{A} \bar{B}^{2}
\end{aligned}
$$

with solution

$$
\bar{A}(u)=\left(\frac{u}{u_{0}}\right)^{1 / d}, \quad \bar{B}(u)=\left(\frac{u_{0}}{u}\right)^{(d-1) / 2 d} e^{\left(u_{0}^{2}-u^{2}\right) / 2 u_{0}}
$$

where

$$
u_{0} \equiv\left(\frac{M_{d+2}}{f}\right)^{d}
$$

For a complete exterior solution these functions must be matched onto a metric valid within the vortex core, determining the parameter $\gamma$, which naturally is of size $\gamma \sim 1 / f$.

Note that our solution has a genuine curvature singularity at $u=0$. (For example, the Laplacian of the Ricci scalar diverges as $\sim u^{-2 / d}$ at $u=0$.) Furthermore, this is a naked singularity, located at a finite proper distance from the string core near $u \sim u_{0}$.

Because of the existence of a naked singularity it is not immediately clear whether or not sensible physical results follow from our solution [19, 20, 21]. There are two different problems that might arise. The first is that the existence of the singularity is not reliable, precisely because gravitational forces become strong there. A higher derivative correction to the Einstein-Hilbert action for example, would probably eliminate - or at least change the nature of the singularity. We will argue later that, while this may indeed happen in extensions of Einstein's theory, it would likely not change the conclusions we arrive at by assuming the singularity to be physical.

A second problem is that the conservation of energy and momentum might be violated at the singularity. This can be phrased as a question concerning the boundary conditions that must be applied to fields at the singularity [22]. Just as the irregular Legendre functions which appear when solving the non-relativistic Schrödinger equation for the hydrogen atom must be discarded, as they correspond to probability loss at the origin, so must any solution here which loses energy or momentum through the singularity. Thus we must search for conditions on fields propagating in the background metric that insure conservation of quantum 
numbers at the singular boundary of the spacetime. The ability to find such conditions is not guaranteed if the singularity is too strong, however. To address this issue we first consider propagation of a massless scalar in our background metric; we then consider gravitational perturbations.

\section{Scalar fields and boundary conditions}

We consider a massless scalar field $\phi$ satisfying the wave equation

$$
\square \phi=\frac{1}{\sqrt{-g}} \partial_{\mu}\left(\sqrt{-g} g^{\mu \nu} \partial_{\nu} \phi\right)=0
$$

It is convenient to introduce a second parameterization of the transverse radial dimension:

$$
d s^{2}=A(z)^{2} \eta_{a b} d x^{a} d x^{b}+A(z)^{2} d z^{2}+\gamma^{2} B(z)^{2} d \theta^{2}
$$

with

$$
z=\gamma \int_{0}^{u} \frac{\bar{B}\left(u^{\prime}\right)}{\bar{A}\left(u^{\prime}\right)} d u^{\prime}, \quad A(z) \equiv \bar{A}(u(z)), \quad B(z) \equiv \bar{B}(u(z)) .
$$

The functions $\bar{A}(u)$ and $\bar{B}(u)$ are the solutions found previously in eq. (6). For small $u$, $z$ behaves like $z \sim u^{(d-1) / 2 d}$, so that the singularity at $u=0$ is also at $z=0$. Near the singularity

$$
A(z) \rightarrow z^{2 /(d-1)}, \quad B(z) \rightarrow \frac{1}{z}
$$

Using the isometries of the background metric, we search for solutions of the form

$$
\phi=e^{i p \cdot x} e^{i n \theta} \frac{\varphi(z)}{\psi_{1}(z)}, \quad \eta_{a b} p^{a} p^{b}=-m^{2}
$$

where $p \cdot x \equiv \eta_{a b} p^{a} x^{b}$ and

$$
\psi_{1}(z)=\left(g^{z z} \sqrt{-g}\right)^{1 / 2}=\left(\gamma A^{d-1} B\right)^{1 / 2} .
$$

The parameter $m$ will be the conventional $d$-dimensional mass of this mode. The function $\varphi(z)$ then satisfies the equation

$$
\left[-\frac{\mathrm{d}^{2}}{\mathrm{~d} z^{2}}+V(z)+n^{2} \frac{A^{2}}{B^{2}}\right] \varphi(z)=m^{2} \varphi(z), \quad V(z)=\frac{\psi_{1}^{\prime \prime}}{\psi_{1}}
$$

This equation can be recast in the more convenient form

$$
\left[\bar{Q} Q+n^{2} \frac{A^{2}}{B^{2}}\right] \varphi(z)=m^{2} \varphi(z)
$$


where

$$
Q=-\frac{\mathrm{d}}{\mathrm{d} z}+\frac{d \log \psi_{1}(z)}{d z}, \quad \bar{Q}=\frac{\mathrm{d}}{\mathrm{d} z}+\frac{d \log \psi_{1}(z)}{d z}
$$

In this form, the scalar wave equation clearly has two zeromode solutions with $n=m=0$. One such solution is easily identified

$$
\varphi(z)=\psi_{1}(z)
$$

The second zeromode follows as

$$
\varphi(z)=\psi_{2}(z) \equiv \psi_{1}(z) \int^{z} \frac{d z^{\prime}}{\psi_{1}\left(z^{\prime}\right)^{2}}=\psi_{1}(z) \log (u(z)) .
$$

Near the singularity at $z=0$ these two solutions behave as

$$
\psi_{1}(z) \rightarrow \sqrt{z}, \quad \psi_{2}(z) \rightarrow \sqrt{z} \log z
$$

Eq. (15) shows that the $m^{2}$ and $n^{2}$ terms become irrelevant near the singularity, and thus all solutions behave similarly to the two zeromode solutions in the limit $z \rightarrow 0$. We will refer to the solutions behaving as $\sqrt{z}$ near $z=0$ as the "regular" solutions, while those behaving as $\sqrt{z} \ln z$ for small $z$ will be called the "irregular" solutions.

The fact that our spacetime is geodesically incomplete should not matter provided that no conserved quantities are allowed to leak out through the boundary. The $d$-dimensional Poincaré isometries of the metric eq. (2), as well as the axial rotation isometry, lead to conservation laws. The Killing vectors corresponding to these isometries are

$$
\begin{aligned}
\xi_{\mu}^{(a)} & =A^{2} \delta_{\mu}^{a} \\
\xi_{\mu}^{(a b)} & =A^{2}\left(\delta_{c}^{a} \delta_{\mu}^{b}-\delta_{\mu}^{a} \delta_{c}^{b}\right) x^{c} \\
\xi_{\mu}^{(\theta)} & =B^{2} \delta_{\mu}^{\theta}
\end{aligned}
$$

with $a, b, c=1 \ldots d$. For each of these Killing vectors we may contract with the stress-energy tensor to form a current

$$
J^{\mu}=T^{\mu \nu} \xi_{\nu}
$$

satisfying a covariant conservation law

$$
\frac{1}{\sqrt{-g}} \partial_{\mu}\left(\sqrt{-g} J^{\mu}\right)=0
$$

Therefore we demand that the flux through the singular boundary of our spacetime must vanish for each of these currents. For example, for the translation isometries this requires

$$
\lim _{z \rightarrow 0} \sqrt{-g} J_{(a)}^{z}=\lim _{z \rightarrow 0} \sqrt{-g} g^{z z} \partial_{a} \phi \partial_{z} \phi=0
$$


Using eq. (19) and the form of $\phi$ eq. (12) we see that the flux through the singularity for the regular solutions vanishes, while that for the irregular solutions diverges. Therefore the boundary conditions boundary conditions we have chosen eliminate the irregular solutions. The other currents lead to the same condition.

Once the irregular solutions have been discarded, it follows that that $\bar{Q}=Q^{\dagger}$; that is, $\left(\varphi_{1}, \bar{Q} \varphi_{2}\right)=\left(Q \varphi_{1}, \varphi_{2}\right)$ where $\varphi_{1,2}$ are arbitrary regular solutions, since the difference between the two expressions is a vanishing surface term. Therefore $\bar{Q} Q$ is a positive semidefinite operator, implying that the $m^{2}$ eigenvalues are all positive, so that no tachyonic modes are seen in the $d$-dimensional world.

In fact the boundary condition eq. (23) (supplemented by appropriate boundary conditions near the string core) is precisely the condition necessary to insure that the eigenvalue problem specified by eqs. (8,12) is of the classic Sturm-Liouville type. Thus not only is the spectrum non-tachyonic, but the eigenfunctions are also complete, discrete, and my be chosen to be orthogonal.

\section{Gravitational perturbations and Newton's law of grav- itation}

We turn to the metric fluctuations about the solution eq. (6). The metric in $(d+2)$ dimensions has $(d+2)(d-1) / 2$ physical polarizations; for a $d$-dimensional observer these decompose into a $d$-dimensional graviton, $2 d$-dimensional vectors and $3 d$-dimensional scalars. We first focus on the $d$-dimensional graviton fluctuations. We consider a perturbation of the metric of the form

$$
d s^{2}=A(z)^{2}\left(\eta_{a b}+h_{a b}\right) d x^{a} d x^{b}+A(z)^{2} d z^{2}+\gamma^{2} B(z)^{2} d \theta^{2}
$$

and impose the gauge conditions $\partial_{a} h^{a b}=h_{a}^{a}=0$, following the analysis of ref. [14. Using the isometries of the background metric we will search for solutions of the form

$$
h_{a b}=\varepsilon_{a b} e^{i p \cdot x} e^{i n \theta} \frac{\varphi(z)}{\psi_{1}(z)}
$$

where $\epsilon_{a b}$ is a constant polarization tensor, and $\psi_{1}$ is the same function defined in eq. (13).

We can already identify one solution of the form eq. (25): the original metric eq. (9) has an invariance under restricted general coordinate transformations along the brane. This is enough to insure the presence of a massless graviton along the brane. The fluctuation in the metric corresponding to this mode may be found by replacing $\eta_{a b} \rightarrow \bar{g}_{a b}$; that is, the wave function of this mode is just an $h_{a b}$ independent of the transverse coordinates:

$$
\varphi(z)=\psi_{1}(z)
$$

\footnotetext{
${ }^{2}$ Note that, strictly speaking, it is the combination $\left(\sqrt{-g} g^{i i}\right)^{1 / 2}$ that appears in the denominator of eq. (25). For our coordinate choice, in which $g^{i i}=g^{z z}=1 / A^{2}$, this equals $\psi_{1}$.
} 
with $n=m=0$.

The coupling of this mode to stress-energy along the brane may be computed by examining the action for this fluctuation. Using eqs. (25, 26) we get an action

$$
S=-M_{d+2}^{d} \int \sqrt{-g} d z d \theta d^{d} x \sqrt{-(\eta+h)} \frac{R_{d}}{A^{2}}
$$

where $R_{d}$ is the $d$-dimensional curvature computed from $(\eta+h)_{a b}$. This allows identification of the $d$-dimensional Planck scale as ${ }^{5}$ :

$$
M_{d}^{d-2}=M_{d+2}^{d} \int \psi_{1}(z)^{2} d z d \theta=e^{u_{0}} u_{0}^{(d+1) / 2 d} \Gamma\left(\frac{d-1}{2 d}\right) \pi \gamma^{2} M_{d+2}^{d} .
$$

This form demonstrates the connection between the gravitational coupling in $d$-dimensions, and the normalization of the zeromode $\psi_{1}$. The factor $e^{u_{0}}$ dominates this expression, providing an exponential hierarchy. For the especially interesting case of $d=4$, with $\gamma=1 / f$, a ratio of $M_{6} / f \simeq 2.7$ yields a hierarchy for $M_{4} / M_{6}$ of over $10^{17}$ !

The Einstein equation for general fluctuations of the form eq. (25) reduces to the scalar wave equation eq. (15) which we considered in the previous section:

$$
\left[\bar{Q} Q+n^{2} \frac{A^{2}}{B^{2}}\right] \varphi(z)=m^{2} \varphi(z)
$$

where $Q$ is the same operator as in the scalar case. Our analysis is therefore identical to that of the previous section: we discard all solutions for which $\varphi(z)$ behaves like $\sqrt{z} \log z$ near the singularity. The remaining solutions include the zeromode, behaving as a massless $d$-dimensional graviton on the branet, and the Kaluza-Klein like modes with non-zero $\mathrm{m}^{2}$. All these modes have $\varphi(z)$ vanishing like $\sqrt{z}$ as we approach the singularity. Since $\bar{Q} Q$ is positive semidefinite, we are assured that the Kaluza-Klein modes all have real masses, and that there are no gravitational instabilities. Furthermore, the spectrum is discrete, with spacing set by

$$
\Delta m^{2} \sim 1 / z_{0}^{2} \sim \frac{\left(M_{d+2}\right)^{d}}{\left(M_{d}\right)^{d-2}}
$$

where $z_{0}=z\left(u_{0}\right)$ and we made use of eq. (28). This is the same relation one expects in the large extra dimension scenario. Since these Kaluza-Klein modes are coupled with gravitational strength, they are still acceptable, even if they are extremely light [5].

As for the other modes of the graviton field, we expect the three scalar modes and one of the vector modes that arise from the decomposition to have masses on the same scale

\footnotetext{
${ }^{3}$ In principle we only trust the integrand in the region outside the string core. However the integral is dominated by this region-inclusion of the string core changes the value of the integral by an exponentially small amount.

${ }^{4}$ Unlike in the scalar case, we are assured that there will be a solution with $m^{2}=0$, since whatever occurs in the core of the vortex will maintain general coordinate invariance.
} 
as $\Delta m$. However, one of the vector modes is corresponds to the rotational isometry of the metric. Normally, one would then expect this field to be the vector potential associated with an unbroken $U(1)$ gauge symmetry in the $d$-dimensional world. However, the scalar field $\Phi$ breaks the rotational symmetry. Therefore this gravi-photon eats the Goldstone boson associated with phase rotations of $\Phi$, and gets a mass ${ }^{2}$ comparable to the $\Delta m^{2}$ of eq. (30).

\section{Conclusions and speculations}

We have shown that the metric outside a global cosmic 3-brane provides a dynamical determination of the hierarchy between the high scale associated with Newton's constant, $10^{19}$ $\mathrm{GeV}$, and the low scale associated with particle physics, a few TeV. With no fine tuning of fundamental parameters, this metric produces a finite, large transverse area. Small fluctuations about this background metric behave as a normal, 4-d graviton along the brane. The coupling of this mode to excitations on the brane, the effective Newton constant, is the 6 -d Newton constant, taken to be characterized by the TeV scalep, divided by the area of the transverse dimensions. Since this area is related to the intrinsic scale by a factor $\sim \exp \left(\left(M_{6} / f\right)^{4}\right)$, a ratio of $M_{6} / f \sim 2.7$ easily provides the necessary hierarchy.

The fact that our metric is geodesically incomplete does not make our solution inconsistent, since we are able to find boundary conditions on our fields that guarantee no conserved quantities are lost through the singularity. Nevertheless, we expect that physics beyond Einstein's theory will alter the nature of the singularity, and possibly eliminate it altogether. Even in the latter case, gravity would become strong, even if not singular, only at an exponentially large distance from the vortex. The key feature of our solution - the dynamical generation of an exponentially large length scale — would remain intact. Furthermore, it is quite plausible that the low lying solutions to the wave equations we have considered will not be appreciably altered, precisely because the boundary conditions we have chosen make the solutions insensitive to what is happening at the singularity. They would be analogous to the low lying quantum mechanical states in a deep square well potential, which are insensitive to whether the potential is actually infinite outside the box, or whether it is finite but large.

In our discussion the nature of the 3-brane played little role. The only requirement was the existence of stress-energy of the form eq. (3). Such a stress-energy tensor applies when scalar field equations have stable brane solutions from spontaneously broken global symmetries (such as a cosmic "string"), or from long range fieldss [24 attached to D-brane?. While we specifically discussed a brane with codimension equal to two, it seems possible that one could find similar solutions with exponentially large extra dimensions starting with branes of other codimensions — preferably a D-brane that could support a realistic 4-d world. It is interesting to speculate whether the winding number of a bulk topological field about this brane could determine the number and chirality of families living on the brane.

\footnotetext{
${ }^{5}$ This scale may need to be more nearly $100 \mathrm{TeV}$ to satisfy all phenomenological constraints [23].

${ }^{6}$ The example of Arkani-Hamed, Dimopolous and March-Russell [25] for obtaining logarithmic running through a massless scalar field coupled to a 3-brane provides another example of a D-brane as a source for a massless scalar field
} 


\section{Acknowledgments}

We thank Nima Arkani-Hamed, Ann Nelson, Lisa Randall, Martin Schmaltz, and Raman Sundrum for useful conversations. We would like to express our gratitude to the Aspen Center for Physics, where this work was begun. A.G.C. is supported in part by DOE grant \#DE-FG02-91ER-40676; D.B.K. is supported in part by DOE grant \#DOE-ER-40561.

\section{References}

[1] T. Kaluza, Preus. Acad. Wiss. K1, 966 (1921).

[2] O. Klein, Zeit. Phys. 37, 895 (1926).

[3] O. Klein, Nature 118, 516 (1926).

[4] I. Antoniadis, Phys. Lett. B246, 377 (1990).

[5] N. Arkani-Hamed, S. Dimopoulos, and G. Dvali, Phys. Lett. B429, 263 (1998), hepph/9803315.

[6] I. Antoniadis, N. Arkani-Hamed, S. Dimopoulos, and G. Dvali, Phys. Lett. B436, 257 (1998), hep-ph/9804398.

[7] R. Sundrum, Phys. Rev. D59, 085010 (1999), hep-ph/9807348.

[8] N. Arkani-Hamed, S. Dimopoulos, and J. March-Russell, (1998), hep-th/9809124.

[9] N. Arkani-Hamed, private communication .

[10] L. Randall and R. Sundrum, (1999), hep-ph/9905221.

[11] W. D. Goldberger and M. B. Wise, (1999), hep-ph/9907447.

[12] O. DeWolfe, D. Z. Freedman, S. S. Gubser, and A. Karch, (1999), hep-th/9909134.

[13] M. Visser, Phys. Lett. 159B, 22 (1985), hep-th/9910093.

[14] L. Randall and R. Sundrum, (1999), hep-th/9906064.

[15] M. Gogberashvili, (1999), hep-ph/9908347.

[16] N. Arkani-Hamed, S. Dimopoulos, G. Dvali, and N. Kaloper, (1999), hep-th/9907209.

[17] J. Lykken and L. Randall, (1999), hep-th/9908076.

[18] A. G. Cohen and D. B. Kaplan, Phys. Lett. B215, 67 (1988).

[19] R. Gregory, Phys. Lett. B215, 663 (1988). 
[20] G. W. Gibbons, M. E. Ortiz, and F. R. Ruiz, Phys. Rev. D39, 1546 (1989).

[21] R. Gregory, Phys. Rev. D54, 4955 (1996), gr-qc/9606002.

[22] M. Gell-Mann and B. Zwiebach, Nucl. Phys. B260, 569 (1985).

[23] L. J. Hall and D. Smith, Phys. Rev. D60, 085008 (1999), hep-ph/9904267.

[24] G. Dvali, Phys. Lett. B459, 489 (1999), hep-ph/9905204.

[25] N. Arkani-Hamed, S. Dimopoulos, and J. March-Russell, (1999), hep-th/9908146. 